\title{
Kinesthetic imagery provides additive benefits to internal visual imagery on slalom task performance
}

Callow, Nichola; Jiang, Dan; Roberts, Ross; Edwards, M.

\section{Journal of Sport and Exercise Psychology}

DOI:

10.1123/jsep.2016-0168

Published: 01/02/2017

Peer reviewed version

Cyswllt i'r cyhoeddiad / Link to publication

Dyfyniad o'r fersiwn a gyhoeddwyd / Citation for published version (APA):

Callow, N., Jiang, D., Roberts, R., \& Edwards, M. (2017). Kinesthetic imagery provides additive benefits to internal visual imagery on slalom task performance. Journal of Sport and Exercise Psychology, 39(1), 81-86. https://doi.org/10.1123/jsep.2016-0168

\section{Hawliau Cyffredinol / General rights}

Copyright and moral rights for the publications made accessible in the public portal are retained by the authors and/or other copyright owners and it is a condition of accessing publications that users recognise and abide by the legal requirements associated with these rights.

- Users may download and print one copy of any publication from the public portal for the purpose of private study or research.

- You may not further distribute the material or use it for any profit-making activity or commercial gain

- You may freely distribute the URL identifying the publication in the public portal ?

Take down policy

If you believe that this document breaches copyright please contact us providing details, and we will remove access to the work immediately and investigate your claim. 
Title: Kinesthetic imagery provides additive benefits to internal visual imagery on slalom task performance

Nichola Callow ${ }^{1}$, Dan Jiang ${ }^{1}$, Ross Roberts ${ }^{1}$, \& Martin G. Edwards ${ }^{2}$

${ }^{1}$ Institute for the Psychology of Elite Performance, Bangor University, UK

${ }^{2}$ Institut de Recherches en Sciences Psychologiques, Université catholique de Louvain, Belgium 


\begin{abstract}
Recent brain imaging research demonstrates that the use of internal visual imagery (IVI) or kinesthetic imagery (KIN) activates common and distinct brain areas. In this paper we argue that combining the imagery modalities (IVI \& KIN) will lead to a greater cognitive representation (with more brain areas activated), and this will cause a greater slalom-based motor performance compared to when using IVI alone. To examine this assertion, we randomly allocated 56 participants to one of three groups: IVI, IVI \& KIN, or a math-control. Participants performed a slalom based driving task in a driving simulator, with average lap time used as a measure of performance. Results revealed the IVI \& KIN group achieved significantly quicker lap times than IVI and the control groups. The discussion includes a theoretical advancement on why the combination of imagery modalities might facilitate performance, with links made to the cognitive neurosciences literature and applied practice.
\end{abstract}


Research examining the effects of imagery on the acquisition and execution of motor performance has delineated imagery into modalities and perspectives. This delineation includes visual and kinesthetic sensory modalities (e.g., Fourkas, Avenanti, Urgesi, \& Aglioti, 2006; Guillot, Collet, Nguyen, Malouin, Richards, \& Doyon, 2009; Hardy \& Callow, 1999) with the visual modality being further separated into two visual imagery perspectives. These two visual perspectives are: external visual imagery perspective (EVI: where the imaginer watches him or herself performing the action from an observer's position; as if watching him or herself on television) and internal visual imagery perspective (IVI: where the imaginer looks out through his or her own eyes while performing the action). The kinesthetic imagery modality (KIN) is defined as how it feels to perform an action, and includes aspects such as the force and effort involved in movement (Callow \& Waters, 2005).

The use of IVI and EVI has been shown to have different impacts on the motor system, with the different perspectives specifically moderating the ability to perform certain tasks. For example, the use of EVI compared to IVI has been shown to produce significant improvements in activities that involve form based movement patterns, such as climbing or gymnastics (Hardy \& Callow, 1999). In slalom line-based activities, such as down-hill slalom skiing, the use of IVI produces more accurate motor performance than EVI (Callow, Roberts, Hardy, Jiang, \& Edwards, 2013; White \& Hardy, 1995). Further, in form-based tasks KIN produces significant performance gains over and above the visual imagery perspective if an individual has expertise in a movement (Hardy \& Callow, 1999). Cognitive explanations for these effects have been proposed (e.g., Hardy, 1997; Callow et al., 2013) where the use of imagery can benefit motor performance by allowing individuals to supplement information that is already available from the physical movement. Specifically, the cognitive representation of KIN (and knowing how it feels to perform the imagined performance; perhaps particularly for skilled individuals) supplements the visual imagery of knowing how it looks to perform the performance. The 
parallel cognitive representations of the two imagery modalities allows for a combined and richer cognitive representation that leads to a greater effect on performance (Hardy, 1997).

This line of reasoning is supported by the cognitive neuroscience imagery literature. There is evidence that the observation or the imagery of actions cause neural activations in similar areas of the brain as those used for executing action (e.g., Guillot et al., 2009). In a recent paper we extended the findings of Guillot et al. (Jiang, Edwards, Mullen, \& Callow, 2015) and demonstrated common hMNS neural activation for the imagery modalities and perspectives of KIN, IVI and EVI, particularly in motor region Brodmann Area 6 (BA6). Interestingly, differential areas were also activated. Specifically, brain activations for IVI subtracted from EVI showed no significant areas of activity, while KIN subtracted from EVI showed that the EVI caused parietal occipital-temporal ventral stream area activations (including the right inferior parietal lobule, BA7; right middle occipital cortex, BA39; and right superior and middle temporal gyrus BA22, BA39). EVI subtracted from IVI, and KIN subtracted from IVI showed similar effects, with the IVI causing significant areas of activity associated with the dorsal stream, extending from the occipital-parietal areas of BA7 to the premotor areas of BA44 and BA6 (i.e., the same areas of the brain associated with the hMNS). Finally, EVI subtracted from KIN showed that the KIN lead to some fontal activity (BA47) and bilateral caudate activation (replicating Guillot et al., 2009). IVI subtracted from KIN showed no additional activity. These findings show that while all imagery modalities and perspectives activate common brain areas there also exist distinct brain areas that are activated during the specific type of imagery. Therefore, linked to the previous paragraph, the combination of imagery modalities would cause more areas of the brain to be activated, and it could be that the combined activations associated with different imagery types may cause a richer cognitive representation of the imaged performance, and that this richer cognitive 
representation may lead to greater performance effects (cf. Holmes \& Collins, 2001; Wakefield, Smith, Aidan, \& Holmes, 2013).

Although it is know that KIN can have an additional performance benefit over and above EVI for form-based tasks (e.g., Hardy \& Callow, 1999), it is not known if KIN in combination to IVI (commonly referred to as motor imagery in the cognitive neuroscience literature) would show additive benefits for slalom-based tasks compared to IVI alone. Consequently, we investigated this possible additive benefit using a slalom-based task. Specifically, we hypothesize that there would be more beneficial effects in the performance of a driving simulator task when combining IVI with KIN than when using IVI alone. We made this hypothesis based on the underlying neuroscientific explanations presented earlier that the IVI and KIN combination should provide a richer cognitive representation (or functional equivalence Jeannerod, 1994, 2001) and more likely to influence subsequent performance than IVI alone. The present study not only has potential to advance our theoretical understanding of imagery, but also as an essential reference for applied practice when using the important psychological skill of imagery with athletes.

\section{Method $^{1}$}

\section{Participants}

We recruited 56 male participants from a UK University’s Sport Science School ( $M$ age $=21.77$ years, $S D=3.20$ ) for the experiment. All participants had held a UK driving license for at least 1 year, and all reported having played computer games less than once per week in the preceding 6 months. Participants provided written informed consent, and the School's Ethics Board granted ethical approval for the study. Of the 56 participants, four did not achieve the set Vividness of Movement Imagery Questionnaire-2 criteria score of equal to or less than 36 for each imagery perspective and modality, indicating moderately clear and vivid imagery 
ability (cf. Roberts, Callow, Hardy, Markland, \& Bringer, 2008). Further, seven participants were screened out due to their responses on the post-experimental questionnaire (see Results). Thus the final sample was $45(M$ age $=21.75$ years, $\mathrm{SD}=3.22)$.

\section{Experimental Apparatus and Task}

The task involved the completion of laps in a simulated rally driving circuit, with the average lap time used as the measure of performance. The driving-simulation slalom task was undertaken in a purpose-built driving simulator, incorporating a rally car seat, a force feedback steering wheel (which could be turned $\pm 900^{\circ}$ to keep the car on the circuit), 6-speed gear shifter and pedals. The driving simulator was connected to a 22 inch LCD monitor displaying the Gran Turismo 5 Prologue game (Codemaster, Warwickshire). The Suzuka Circuit and Eiger Nordward circuit were used in training and experimental phase of the experiment respectively. These circuits were used because they have, for example, quadruple-hairpin switchback sections, corners and a number of significant and severe camber and gradient changes. Thus the participants had to make movement changes based on precise spatial and temporal locations as per a slalom task. The virtual reality display presented the driver's view out through the front window of the car as if actually driving the car.

\section{Training and Experimental Phases}

Participants completed a 90-min training phase period where they had to achieve two criteria (derived from pilot testing). These criteria were the completion of three consecutive laps under 170 s, and a plateau in performance, indicated by the last three lap times falling within 5s of each other (cf. Wilson, Chattington, Marple Horvat, \& Smith, 2007). If participants achieved the criteria, they then proceeded to the experimental phase. In the experimental phase, participants completed a total of 15 laps (five practice, five pre-imagery, five post-imagery) of 
the simulated rally driving circuit, with average lap time at pre and post-imagery condition used as the measure of performance.

\section{Experimental Groups and Imagery Scripts ${ }^{2}$}

The 56 participants were randomly assigned to one of three groups; internal visual imagery (IVI), an internal visual imagery combined with kinesthetic imagery (IVI \& KIN), or math-control. Following practice and pre-imagery performance measures, participants in the imagery groups listened to an audio recording of an imagery script pertaining to the imagery group to which they were allocated. Scripts were developed using Lang's (1984) guidelines for including stimulus, response and meaning propositions and pilot tested prior to data collection. Further, the IVI script detailed the task from a first person visual perspective, requiring the participants to image the task through his/her own eyes. The IVI \& KIN script detailed the task from the same visual perspective, but also included all the physical feelings involved in driving. In order to maintain experimental control, although the scripts were developed by the authors, there was flexibility in the scripts (e.g., participants were asked to imagine their view change as they turned a corner). This flexibility allows participants to develop their own images, thus providing a degree of individualization, and consequently the images being meaningful for the participants (cf. Wilson, Smith, Burden, \& Holmes, 2010). The scripts took $\sim 120 \mathrm{~s}$ to administer. In the math-control condition, participants were required to answer standard arithmetic questions (e.g., $14+4+6$ ).

\section{Measures}

Time-taken to complete each lap was measured automatically (in seconds) by the Gran Turismo 5 Prologue software, and recorded by the experimenter. Note that the line of driving moderated the time, with cutting corners reducing the time compared to driving in the center of the road, but with collisions with curbs, or driving on the grass adding to the time. 
To determine participant imagery ability they completed the Vividness of Movement Imagery Questionnaire-2 (VMIQ-2: Roberts et al., 2008). The VMIQ-2 has 12 items repeated on three subscales (EVI, IVI, and KIN), and is measured on a 5-point Likert scale ranging from 1(perfect clear and as normal as perfect vision/feel of the movement) to 5(no image at all, you only know that you are thinking of the skill. The VMIQ-2 has demonstrated acceptable factorial validity, construct validity and concurrent validity (see Roberts et al., 2008). For the present study Cronbach Alpha's of the EVI, IVI, and KIN VMIQ-2 subscales were $\alpha=.86, \alpha=.90$ and $\alpha=.91$ respectively.

On completion of all trials, we also administered a manipulation check questionnaire. The first question asked all participants whether they had been able to adhere to the treatment group. The remaining questions were only given to participants in the two imagery groups, and they asked whether the participant had experienced any switching of visual imagery perspectives during the task, and whether and to what extent they had experienced any kinesthetic imagery during their use of visual imagery.

\section{Procedure}

One week prior to the experiment, participants completed the VMIQ-2. Participants attended the laboratory individually and received instructions about the purpose of the experiment. Participants then completed the 90 min training phase. After 15 min break, all the participants completed the experimental phase, and then the manipulation check questionnaire.

\section{Results}

\section{Data Screening and Preliminary Analyses}

In addition to the four participant who did not achieve the imagery ability criteria, the post-experimental questionnaire revealed that seven participants either switched between

modalities or did not adhere to their treatment group (e.g., scoring below 6 for kinesthetic 
imagery experience when in the IVI \& KIN group). In terms of demographic variables and imagery ability we visually inspected and compared the mean data of the seven participants who were excluded against the mean data of the remaining 45 participants. We could see no discernable differences, and thus conducted the planned analyses on the data from the remaining 45 participants.

All assumptions were met for the different analyses. Separate one-way ANOVAs revealed no differences in imagery ability between the three groups for IVI imagery ability $F$ $(2,42)=0.46, p=0.63$, EVI imagery ability $F(2,42)=1.29, p=0.29$ and kinesthetic imagery ability $F(2,42)=0.83, p=0.45$. A t-test on the manipulation check question related to kinesthetic imagery experience revealed a significant difference in kinesthetic imagery experience between the IVI and IVI \& KIN groups $t(2,28)=-10.36, p<0.01$, with the mean data revealing, as expected, higher kinesthetic imagery experience for the IVI \& KIN group. See Table 1 for mean and standard deviations for imagery ability and kinesthetic experience.

\section{Performance Score (time-taken)}

A mixed-model (group $\times$ test; pre verses post) ANOVA indicated no significant effect for group, a significant main effect for test, $F(1,42)=75.14, p<.001$, partial $\eta^{2}=.64, d=0.41$ and a significant group by test interaction, $F(2,42)=22.30, p<.001$, partial $\eta^{2}=.52$. Tukey's corrected post-hoc tests for the interaction revealed a significant improvement in performance (indicating a quicker time) from pre to post test for the IVI group $F(1,14)=22.28, p<.001$, $d=0.46$ and the IVI \& KIN group $F(1,14)=86.36, p<.001, d=0.84$. There was no change in performance for the math-control group $F(1,14)=0.01, p=.94, d=0.01$. In addition, there were no significant differences between groups at pre-test. However, at post-test, performance was significantly quicker for the IVI \& KIN compared to the math-control, $q(42)=13.06, p<$ .001 , and the IVI group, $q(42)=10.75, p<.001$. There was no significant difference between 
the IVI group and the math-control group at post-test. Given this latter result, coupled with the significant difference pre to post test for the IVI group, but not the math-control group, we ran an additional Tukey's test on the change scores for these two groups. Performance improvement was significantly greater for the IVI than the math-control group $q(42)=7.64, p$ $<.001 d=0.43$. See Table 1 and Figure 1 for time-taken descriptives at pre and post-test.

\section{Discussion}

The aim of the present study was to test the hypothesis that there would be more beneficial effects in a driving simulator slalom-based task when combining IVI with KIN than using IVI alone. The results of the experiment replicated Callow et al., (2013) showing that IVI increased performance for post-imagery compared to pre-imagery performance, whereas there was no change in performance for the control group. Furthermore, our hypothesis that the addition of KIN to IVI would cause an enhancement in performance relative to IVI alone was supported by the results.

This main finding was robust despite the relatively small samples of participants tested. The main factor contributing to the significant effects was probably the fact that participants already had experience of the task, with all of the participants tested having a UK driving license for at least 1 year. Furthermore, within the study, all of the participants were trained to achieve an error-less and consistent performance. Both of these factors are related to the notion that performers had experience of the task and had the ability to perceive the kinesthetic components associated to actually performing the driving simulator task. As we discussed in the introduction, experience of the performance may be critical for these effects (Hardy \& Callow, 1999).

Additional experimentation could investigate the neurological activations associated with IVI and KIN combined in order to understand the mechanism for the additive benefits of 
KIN and IVI in combination on performance. Theoretically, we propose two plausible mechanisms. One is that IVI and KIN activate independent areas of the brain, and perhaps the two independent areas being activated caused a double-priming effect. Another is that the independent IVI and KIN brain area activations cause a cumulative increase of brain activity in the common hMNS area (particularly BA6) in comparison to IVI or EVI brain activation alone. Therefore, in the first possibility, the enhanced performance is caused by more brain activity perhaps suggesting a richer cognitive representation of the imaged performance, whereas the second possibility is that there is specific increased brain activity in the area associated with action execution (i.e., the brain area of functionally equivalence).

These results provide an interesting first paper to demonstrate the additive benefits of IVI and KIN on performance facilitation. We propose that these findings should be replicated in other slalom-based tasks, including tasks that use more ecologically valid tasks than the laboratory simulation task used in the present study. It could also be useful to evaluate how other tasks dependent upon a first-person, or body centered perspectives benefit from added use of the two modalities of imagery on performance where dynamic kinesthesis is less relevant, for example with Olympic trap shooting. We propose that the main explanation of these effects is caused by richer cognitive representations activating more areas of the brain (and perhaps linked to functional equivalence), it would therefore be interesting to show no differences in the use of IVI and KIN compared to IVI alone in tasks where kinesthesis is less relevant. Conversely in tasks where kinesthesis is relevant (for both slalom and form-based tasks), it would be relevant to establish the level of task experience needed in order for KIN to become effective.

From an applied perspective, the findings here advocate the use of IVI and KIN for moderating sport performance where the task involves correct changes to temporal and spatial locations for successful performance. Further the present findings reinforce the importance of 
developing kinesthetic imagery ability (cf. Hardy \& Callow, 1999), with methods such as layered stimulus response training (e.g., Williams, Cooley, \& Cumming, 2013) providing a mechanism for achieving this. The findings also have the potential to explain inconsistencies in the rehabilitation literature. Specifically, despite the use of motor imagery (IVI \& KIN) by stroke patients there remains some debate about its utility (Ietswaart, Johnston, Dijkerman, Joice, Scott, MacWalter, \& Hamilton, 2011). However across this literature, researchers have often failed to delineate the visual imagery perspective, and motor experience of the patient which are perhaps reasons for the inconsistent findings. Based on the arguments above, and from the discussion of Hardy and Callow (1999), we propose that patients should have had at some time, experience in performing the imaged actions in order for motor imagery to have a possibility of an improvement on performance. Further, imagery may only be effective when the relevant imagery pathway is intact. We propose that studies in neuropsychology should consider these points when testing the effectiveness of motor imagery on rehabilitation.

In summary, the results of the current experiment provide the first evidence that the addition of KIN to IVI provides more beneficial effects over the IVI perspective alone on the performance of slalom-based task, with theoretical advancement provided. Further, the paper provides an essential reference for applied practitioners intervening with imagery. Future research should employ fMRI to explore the brain activation subserving these different imagery perspectives and modalities to extend our understanding of the mechanism for the additive benefits of KIN and IVI on performance not only in sport but across other domains such as rehabilitation. 


\section{References}

Callow, N., Roberts, R., Hardy, L., Jiang, D., \& Edwards, M. G. (2013). Performance improvements from imagery: Evidence that internal visual imagery is superior to external visual imagery for slalom performance. Frontiers in Human Neuroscience, 7 , 697. doi:10.3389/fnhum.2013.00697

Callow, N., \& Waters, A. (2005). The effect of kinesthetic imagery on the sport confidence of flat-race horse jockeys. Psychology of Sport and Exercise, 6, 443-459. doi:10.1016/j.psychsport.2004.08.001

Fourkas, A., Avenanti, A., Urgesi, C., \& Aglioti, S. (2006). Corticospinal facilitation during first and third person imagery. Experimental Brain Research, 168, 143-151. doi:10.1007/s00221-005-0076-0

Goginsky, A. M. \& Collins, D. (1996). Research design and mental practice. Journal of Sports Science, 14, 381-92. doi:10.1080/02640419608727725

Guillot, A., Collet, C., Nguyen, V. A., Malouin, F., Richards, C., \& Doyon, J. (2009). Brain activity during visual versus kinesthetic imagery: An fMRI study. Human Brain Mapping, 30, 2157-2172. doi:10.1002/hbm.20658

Hardy, L. (1997).The Coleman Roberts Griffith address: three myths about applied consultancy work. Journal Applied Sport Psychology, 9, 277-294. doi:10.1080/10413209708406487

Hardy, L., \& Callow, N. (1999). Efficacy of external and internal visual imagery perspectives for the enhancement of performance on tasks in which form is important. Journal of Sport \& Exercise Psychology, 21, 95-112. doi: 10.1123/jsep.21.2.95

Holmes, P. S., \& Collins, D. J. (2001). The PETTLEP approach to motor imagery: A functional equivalence model for sport psychologists. Journal of Applied Sport Psychology, 13, 60-83. doi:10.1080/10413200109339004

Ietswaart, M., Johnston, M., Dijkerman, H. C., Joice, S., Scott, C. L., MacWalter, R. S., \& Hamilton, S. J. C. (2011). Mental practice with motor imagery in stroke recovery: Randomized controlled trial of efficacy. Brain, 134, 1373-1386. doi:10.1093/brain/awr077

Jiang, D., Edwards, M. G. Mullins, P., \& Callow, N., (2015). The neural substrates for the different modalities of movement imagery. Brain and Cognition, 97, 22-31. doi:10.1016/j.bandc.2015.04.005

Jeannerod, M. (1994). The representing brain: Neural correlates of motor intention and imagery. Behavioral and Brain Sciences, 17, 187-201. doi: 10.1017/S0140525X00034026 
Jeannerod, M. (2001). Neural simulation of action: A unifying mechanism for motor cognition. Neuroimage, 14, 103-109. doi:10.1006/nimg.2001.0832

Lang, P.J. (1984). Cognitions in emotion: concept and action. In C.E.Izard, J.Kagan, and R.B. Zajonc (Eds.), Emotions, cognition and behavior (pp. 192-226). New York, NY: University of Cambridge.

Roberts, R., Callow, N., Hardy, L., Markland, D., \& Bringer, J. (2008). Movement imagery ability: Development and assessment of a revised version of the vividness of movement imagery questionnaire. Journal of Sport \& Exercise Psychology, 30, 200-221. doi:10.1123/jsep.30.2.200

Wakefield, C., Smith, D., Aidan, P. M., \& Holmes, P. (2013). Functional equivalence or behavioural matching? A critical reflection on 15 years of research using the PETTLEP model of motor imagery. International Review of Sport and Exercise Psychology, 6,105-121. doi:10.1080/1750984X.2012.724437

White, A., \& Hardy, L. (1995). Use of different imagery perspectives on the learning and performance of different motor skills. British Journal of Psychology, 86, 169-180. doi: 10.1111/j.2044- 8295.1995.tb02554.x

Williams, S. E., Cooley, S. J., \& Cumming, J. (2013). Layered stimulus response training improves motor imagery ability and movement execution. Journal of Sport \& Exercise Psychology, 35, 60-71. doi:10.1123/jsep.35.1.60

Wilson, M., Chattington, M., Marple Horvat, D. E., \& Smith, N. C. (2007). A comparison of self-focus versus attentional explanations of choking. Journal of Sport \& Exercise Psychology, 29, 439-456. doi: 10.1123/jsep.29.4.439

Wilson, C., Smith, D., Burden, A., \& Holmes, P. (2010). Participant-generated imagery Scripts produce greater EMG activity an imagery ability. European Journal of Sports Sciences. 10, 417-425. doi: 10.1080/17461391003770491 


\section{Footnotes}

${ }^{1}$ The imagery research literature has previously been criticized due to a lack of experimental replication (cf. Goginsky, \& Collins, 1996). In order to overcome this criticism we purposefully replicated Study 1 from Callow et al. (2013) with an IVI \& KIN group rather than an EVI group.

${ }^{2}$ Scripts can be obtained on request from the first author. 
89

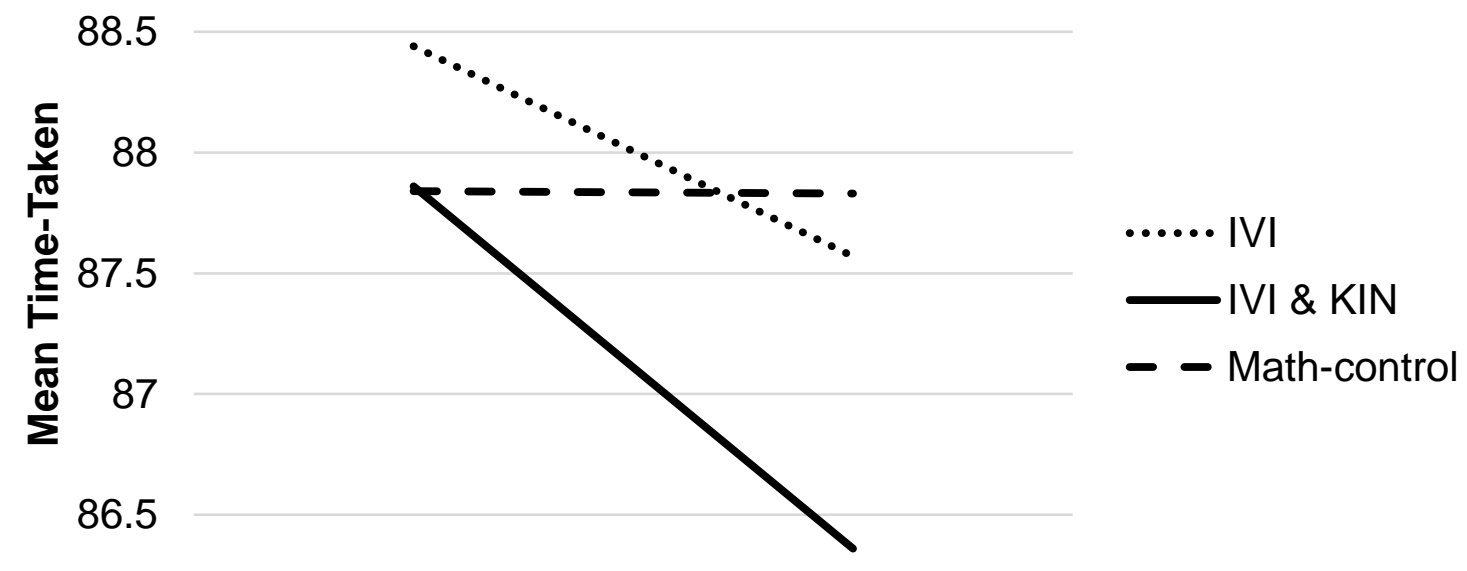

86

Pre-Test

Post-Test

Figure 1. Mean time-taken at pre and post-test. 
Table 1: Means (standard deviations) for VMIQ-2, Kinesthetic Experience and Time-taken at Pre and Post-test

\begin{tabular}{lcccccc}
\hline & \multicolumn{3}{c}{ VMIQ-2 } & $\begin{array}{c}\text { Kinesthetic } \\
\text { Experience }\end{array}$ & Time-taken \\
\cline { 2 - 7 } Group & IVI & EVI & KIN & & Pre-test & Post-test \\
& $\mathrm{n}=15$ & $\mathrm{n}=15$ & $\mathrm{n}=15$ & & 88.44 & 87.57 \\
\hline IVI & 23.29 & 22.14 & 23.36 & 1.79 & $(2.14)$ & $(1.76)$ \\
& $(7.34)$ & $(7.70)$ & $(6.12)$ & $(1.53)$ & & \\
IVI \& KIN & 27.53 & 22.73 & 23.94 & 7.27 & 87.86 & 86.36 \\
& $(5.67)$ & $(5.51)$ & $(7.70)$ & $(1.33)$ & $(2.00)$ & $(1.69)$ \\
\multirow{2}{*}{ Math-control } & 26.27 & 24.80 & 26.67 & - & 87.84 & 87.83 \\
& $(7.53)$ & $(8.87)$ & $(8.00)$ & & $(2.02)$ & $(2.14)$ \\
\hline
\end{tabular}

